\title{
Local Convergence for a Fifth Order Traub-Steffensen-Chebyshev-Like Composition Free of Derivatives in Banach Space
}

\author{
Ioannis K. Argyros ${ }^{1, *}$ and Santhosh George ${ }^{2}$ \\ ${ }^{1}$ Department of Mathematical Sciences, Cameron University, Lawton, OK 73505, \\ USA \\ ${ }^{2}$ Department of Mathematical and Computational Sciences, NIT Karnataka, \\ India 575025
}

Received 13 September 2016; Accepted (in revised version) 24 March 2017

\begin{abstract}
We present the local convergence analysis of a fifth order Traub-SteffensenChebyshev-like composition for solving nonlinear equations in Banach spaces. In earlier studies, hypotheses on the Fréchet derivative up to the fifth order of the operator under consideration is used to prove the convergence order of the method although only divided differences of order one appear in the method. That restricts the applicability of the method. In this paper, we extended the applicability of the fifth order TraubSteffensen-Chebyshev-like composition without using hypotheses on the derivatives of the operator involved. Our convergence conditions are weaker than the conditions used in earlier studies. Numerical examples where earlier results cannot apply to solve equations but our results can apply are also given in this study.
\end{abstract}

AMS subject classifications: 65D10, 49M15, 74G20, 41A25

Key words: Traub-Steffensen-Chebyshev-like composition, restricted convergence domain, radius of convergence, local convergence.

\section{Introduction}

We are concerned with the problem of approximating a solution $x^{*}$ of the equation

$$
F(x)=0,
$$

where $F: D \subseteq \mathscr{B}_{1} \longrightarrow \mathscr{B}_{2}$ is a Fréchet differentiable operator between Banach spaces $\mathscr{B}_{1}, \mathscr{B}_{2}$. Most of the solution methods for solving (1.1) are iterative and for iterative methods order of convergence is an important issue. Convergence analysis of higher order iterative methods require assumptions on the higher order Fréchet derivatives of the operator $F$. That restricts the applicability of these methods. In this study, we consider the local

\footnotetext{
${ }^{*}$ Corresponding author. Email addresses: iargyros@cameron.edu (I. K. Argyros), sgeorge@nitk.ac.in
} (S. George) 
convergence for fifth order Traub-Steffensen-Chebyshev-like composition free of derivatives in Banach space studied by Sharma and Kumar in [16]. The method is defined by

$$
\begin{aligned}
& y_{n}=x_{n}-A_{n}^{-1} F\left(x_{n}\right), \\
& z_{n}=y_{n}-A_{n}^{-1} F\left(y_{n}\right), \\
& x_{n+1}=z_{n}-B_{n} A_{n}^{-1} F\left(z_{n}\right),
\end{aligned}
$$

where

$$
\begin{aligned}
& A_{n}=\left[w_{n}, x_{n} ; F\right], \\
& B_{n}=2 I-A_{n}^{-1}\left[z_{n}, y_{n} ; F\right], \\
& w_{n}=x_{n}+\beta F\left(x_{n}\right),
\end{aligned}
$$

and $[., . ; F]$ is a divided difference of order one on $D^{2}$.

Throughout this paper $L\left(\mathscr{B}_{2}, \mathscr{B}_{1}\right)$ denotes the set of bounded linear operators between $\mathscr{B}_{1}$ and $\mathscr{B}_{2}$ and $B(z, \rho), \bar{B}(z, \rho)$ stand, respectively for the open and closed balls in $\mathscr{B}_{1}$ with center $z \in \mathscr{B}_{1}$ and of radius $\rho>0$.

The motivations for the construction of this method are that is derivative free, of convergence order five and efficient. Notice that in [16] favorable comparisons with other methods using similar information have been provided to show the advantages of the proposed method. The aim of this paper is not to present those comparisons but to extend the applicabilty of method (1.2) in the more general setting of a Banach space. We refer the reader to [16] for more detailed advantages, motivations and comparisons.

Convergence analysis in [16] is based on Taylor expansions and assumptions on the Fréchet derivative $F$ up to the order five when $\mathscr{B}_{1}=\mathscr{B}_{2}=\mathbb{R}^{i}$. That limits the applicability of this method. As a motivational example, let us define function $F$ on $I=\left[-\frac{\pi}{2}, \frac{\pi}{2}\right]$ by

$$
F(x)= \begin{cases}x^{5} \sin \frac{1}{x}+x^{2}+x, & x \neq 0 \\ 0, & x=0 .\end{cases}
$$

Then, $x^{*}=0$, is a solution of $F(x)=0$. We have that

$$
\begin{aligned}
& F^{\prime}(x)=5 x^{4} \sin \frac{1}{x}-x^{3} \sin \frac{1}{x}+2 x+1, \quad F^{\prime}(0)=1, \\
& F^{\prime \prime}(x)=20 x^{3} \sin \frac{1}{x}-5 x^{2} \cos \frac{1}{x}-3 x^{2} \cos \frac{1}{x}-x \sin \frac{1}{x}+2, \\
& F^{\prime \prime \prime}(x)=60 x^{2} \sin \frac{1}{x}-36 x \cos \frac{1}{x}+\sin \frac{1}{x}-\frac{1}{x} \cos \frac{1}{x} .
\end{aligned}
$$

Then, obviously function $F$ does not have bounded third derivative in $I$. In this study we use only assumptions on the first Fréchet derivative of the operator $F$ in our convergence analysis, so that the method (1.2) can be applied to solve equations but the earlier results cannot be applied [1-18] (see Example 3.2). Moreover, we avoid the usage of high order derivatives, since we rely on the computational and approximate computational order of 
convergence that do not require high order derivatives (see Remark 2.2). Notice that finding the radius of convergence is important because it shows the difficulty in obtaining close enough initial points, which otherwise constitute a "shot in the dark". Our new tools of Lipschitz-type conditions allow us to compute a radius of convergence and computable error bounds not provided in [16]. This technique can be used on other iterative methods [9-11, 16-18].

The rest of the paper is structured as follows. In Section 2 we present the local convergence analysis of the method (1.2). We also provide a radius of convergence, computable error bounds and a uniqueness result. Numerical examples are given in the concluding Section 3.

\section{Local convergence}

It is convenient for the local convergence analysis of method (1.2) to define some parameter and scalar functions. Let $\beta \in \mathbb{R}$ be a parameter and let $\omega_{0}:[0,+\infty)^{2} \rightarrow$ $[0,+\infty)$ be a function continuous nondecreasing with $\omega_{0}(0,0)=0$. Define parameter $r_{0}$ by

$$
r_{0}=\sup \left\{t \geq 0: \omega_{0}(\delta t, t)<1\right\} .
$$

Let $v:\left[0, r_{0}\right) \rightarrow[0,+\infty), \omega_{1}:\left[0, r_{0}\right)^{2} \rightarrow[0,+\infty)$ be continuous and nondecreasing functions. Define functions $g_{1}$ and $h_{1}$ on the interval $\left[0, r_{0}\right)$ by

$$
\begin{aligned}
& g_{1}(t)=\frac{\omega_{1}\left(|\beta| v_{0}(t) t, t\right)}{1-\omega_{0}(\delta t, t)}, \\
& h_{1}(t)=g_{1}(t)-1 .
\end{aligned}
$$

Suppose that

$$
\omega_{1}(0,0)<1
$$

Using (2.1) and (2.2), we get that $h_{1}(0)=\frac{\omega_{1}(0,0)}{1-\omega_{0}(0,0)}-1<0$ and $h_{1}(t) \rightarrow+\infty$ as $t \rightarrow r_{0}^{-}$. Then, by intermediate value theorem equation $h_{1}(t)=0$ has solutions in $\left(0, r_{0}\right)$. Denote by $r_{1}$ the smallest such solution. Let $v:\left[0, r_{0}\right) \longrightarrow[0,+\infty)$ be a continuous and nondecreasing function. Define functions $g_{2}$ and $h_{2}$ on the interval $\left[0, r_{0}\right.$ ) by

$$
g_{2}(t)=\left(1+\frac{v\left(g_{1}(t) t\right)}{1-\omega(\delta t, t)}\right) g_{1}(t), \quad h_{2}(t)=g_{2}(t)-1
$$

Suppose that

$$
(1+v(0)) \omega_{1}(0,0)<1 .
$$

We get that $h_{2}(0)<0$ and $h_{2}(t) \rightarrow+\infty$ as $t \rightarrow r_{0}^{-}$. Denote by $r_{2}$ the smallest solution of equation $h_{2}(t)=0$ in the interval $\left(0, r_{0}\right)$. Let $\omega:\left[0, r_{0}\right)^{2} \rightarrow[0,+\infty)$ be a continuous and 
nondecreasing function. Define functions $b, g_{3}$ and $h_{3}$ on the interval $\left[0, r_{0}\right)$ by

$$
\begin{aligned}
& b(t)=\frac{1+\omega\left(\left(\delta+g_{2}(t)\right) t,\left(1+g_{1}(t)\right) t\right)}{1-\omega_{0}(\delta t, t)}, \\
& g_{3}(t)=\left(1+\frac{b(t) v\left(g_{2}(t) t\right)}{1-\omega_{0}(\delta t, t)}\right) g_{2}(t), \\
& h_{3}(t)=g_{3}(t)-1 .
\end{aligned}
$$

Suppose that

$$
(1+(1+\omega(0,0)) v(0))(1+v(0)) \omega_{1}(0,0)<1 .
$$

We obtain by (2.5) that $h_{3}(0)<0$ and $h_{3}(t) \rightarrow+\infty$ as $t \rightarrow r_{0}^{-}$. Denote by $r_{3}$ the smallest solution of equation $h_{3}(t)=0$ in the interval $\left(0, r_{0}\right)$. Define the radius of convergence $r$ by

$$
r=\min \left\{r_{i}\right\}, \quad i=1,2,3 .
$$

Then, for each $t \in[0, r)$ we have:

$$
\begin{aligned}
& 0 \leq g_{i}(t)<1, \\
& 0 \leq \omega_{0}(\delta t, t)<1 .
\end{aligned}
$$

Finally define parameter $R^{*}$ by

$$
R^{*}=\max \{r, \delta r\}
$$

Next, the local convergence analysis of method (1.2) is presented.

Theorem 2.1. Let $F: \Omega \subset \mathscr{B}_{1} \rightarrow \mathscr{B}_{2}$ be a continuous operator and let [.,.;F]: $\Omega^{2} \rightarrow$ $L\left(\mathscr{B}_{1}, \mathscr{B}_{2}\right)$ be a divided difference of order one on $\Omega^{2}$ for $F$. Suppose: there exists $x^{*} \in \Omega$ and function $\omega_{0}:[0,+\infty)^{2} \rightarrow[0,+\infty)$ continuous and nondecreasing such that for each $x, y \in \Omega$,

$$
\begin{aligned}
& F\left(x^{*}\right)=0, \quad F^{\prime}\left(x^{*}\right)^{-1} \in L\left(\mathscr{B}_{2}, \mathscr{B}_{1}\right), \\
& \left\|F^{\prime}\left(x^{*}\right)^{-1}\left([x, y ; F]-F\left(x^{*}\right)\right)\right\| \leq \omega_{0}\left(\left\|x-x^{*}\right\|,\left\|y-x^{*}\right\|\right) .
\end{aligned}
$$

Let $\Omega_{0}=\Omega \cap B\left(x^{*}, r_{0}\right)$. There exist $\delta \geq 0$ functions $v, v_{0}:\left[0, r_{0}\right) \rightarrow[0,+\infty), \omega, \omega_{1}$ : $\left[0, r_{0}\right)^{2} \rightarrow[0,+\infty)$, such that for each $x, y, z, u \in \Omega_{0}$

$$
\begin{aligned}
& \left\|I+\left[x, x^{*} ; F\right]\right\| \leq \delta, \\
& \left\|\left[x, x^{*} ; F\right]\right\| \leq v_{0}\left(\left\|x-x^{*}\right\|\right), \\
& \left\|F^{\prime}\left(x^{*}\right)^{-1}\left[x, x^{*} ; F\right]\right\| \leq v\left(\left\|x-x^{*}\right\|\right), \\
& \left\|F^{\prime}\left(x^{*}\right)^{-1}\left([x, y ; F]-\left[y, x^{*} ; F\right]\right)\right\| \leq \omega_{1}\left(\|x-y\|,\left\|y-x^{*}\right\|\right), \\
& \left\|F^{\prime}\left(x^{*}\right)^{-1}([x, y ; F]-[z, u ; F])\right\| \leq \omega(\|x-z\|,\|y-u\|), \\
& \bar{B}\left(x^{*}, R^{*}\right) \subseteq \Omega,
\end{aligned}
$$


and (2.5) holds, where $r_{0}, r, R^{*}$ are defined by (2.1), (2.6) and (2.8) respectively. Then, the sequence $\left\{x_{n}\right\}$ generated for $x_{0} \in B\left(x^{*}, r\right)-\left\{x^{*}\right\}$ by method (1.2) is well defined, remains in $B\left(x^{*}, r\right)$ for each $n=0,1, \cdots$ and converges to $x^{*}$. Moreover, the following estimates hold

$$
\begin{aligned}
& \left\|y_{n}-x^{*}\right\| \leq g_{1}\left(\left\|x_{n}-x^{*}\right\|\right)\left\|x_{n}-x^{*}\right\| \leq\left\|x_{n}-x^{*}\right\|<r \\
& \left\|z_{n}-x^{*}\right\| \leq g_{2}\left(\left\|x_{n}-x^{*}\right\|\right)\left\|x_{n}-x^{*}\right\| \leq\left\|x_{n}-x^{*}\right\| \\
& \left\|x_{n+1}-x^{*}\right\| \leq g_{3}\left(\left\|x_{n}-x^{*}\right\|\right)\left\|x_{n}-x^{*}\right\| \leq\left\|x_{n}-x^{*}\right\|
\end{aligned}
$$

where the functions $g_{i}, i=1,2,3$ are defined previously. Furthermore, if there exists for $R_{1} \geq r$ such that

$$
\omega_{0}\left(R_{1}, 0\right)<1 \text { or } \omega_{0}\left(0, R_{1}\right)<1 \text {, }
$$

then the limit point $x^{*}$ is the only solution of equation $F(x)=0$ in $\Omega_{1}:=\Omega \cap B\left(x^{*}, R_{1}\right)$.

Proof. Estimates (2.11a)-(2.11c) are shown using induction on $k$. We shall show $A_{0}^{-1} \in$ $L\left(\mathscr{B}_{2}, \mathscr{B}_{1}\right)$ which implies $y_{0}, z_{0}$ and $x_{1}$ are well defined by method (1.2) for $n=0$. Using (2.1), (2.6), (2.7b), (2.8), (2.9b) and (2.10a), we have in turn that

$$
\begin{aligned}
& \left\|F^{\prime}\left(x^{*}\right)^{-1}\left(\left[w_{0}, x_{0} ; F\right]-F^{\prime}\left(x^{*}\right)\right)\right\| \\
\leq & \omega_{0}\left(\delta\left\|x_{0}-x^{*}\right\|,\left\|x_{0}-x^{*}\right\|\right) \\
\leq & \left.\omega_{0}\left(\delta \| x_{0}-x^{*}\right)\|,\| x_{0}-x^{*} \|\right) \\
\leq & \omega_{0}(\delta r, r) \leq \omega_{0}\left(\delta r_{0}, r_{0}\right)<1
\end{aligned}
$$

where we also used

$$
\left\|w_{0}-x^{*}\right\|=\left\|\left(I+\beta\left[x_{0}, x^{*} ; F\right]\right)\left(x_{0}-x^{*}\right)\right\| \leq \delta\left\|x_{0}-x^{*}\right\| .
$$

By the Banach perturbation Lemma $[2,8]$ and (2.13), we get that $A_{0}^{-1} \in L\left(\mathscr{B}_{2}, \mathscr{B}_{1}\right)$ and

$$
\left\|A_{0}^{-1} F^{\prime}\left(x^{*}\right)\right\| \leq \frac{1}{1-\omega_{0}\left(\delta\left\|x_{0}-x^{*}\right\|,\left\|x_{0}-x^{*}\right\|\right)} .
$$

Using the first substep of method (1.2) for $n=0$, (2.6), (2.7a) (for $i=1),(2.10 \mathrm{~b}),(2.10 \mathrm{~d}$ ) and (2.14), we get in turn that

$$
\begin{aligned}
\left\|y_{0}-x^{*}\right\| & =\left\|x_{0}-x^{*}-A_{0}^{-1} F^{\prime}\left(x_{0}\right)\right\| \\
& =\left\|\left[A_{0}^{-1} F^{\prime}\left(x^{*}\right)\right] F^{\prime}\left(x^{*}\right)^{-1}\left(\left[w_{0}, x_{0} ; F\right]-\left[x_{0}, x^{*} ; F\right]\right)\left(x_{0}-x^{*}\right)\right\| \\
& \leq \frac{\omega_{1}\left(\left\|w_{0}-x_{0}\right\|,\left\|x_{0}-x^{*}\right\|\right)\left\|x_{0}-x^{*}\right\|}{1-\omega_{0}\left(\delta\left\|x_{0}-x^{*}\right\|,\left\|x_{0}-x^{*}\right\|\right)} \\
& \leq \frac{\omega_{1}\left(|\beta| v_{0}\left(\left\|x_{0}-x^{*}\right\|\right)\left\|x_{0}-x^{*}\right\|,\left\|x_{0}-x^{*}\right\|\right)\left\|x_{0}-x^{*}\right\|}{1-\omega_{0}\left(\delta\left\|x_{0}-x^{*}\right\|,\left\|x_{0}-x^{*}\right\|\right)} \\
& =g_{1}\left(\left\|x_{0}-x^{*}\right\|\right)\left\|x_{0}-x^{*}\right\| \leq\left\|x_{0}-x^{*}\right\|<r,
\end{aligned}
$$


which shows (2.11a) for $n=0$ and $y_{0} \in B\left(x^{*}, r\right)$. Then, by the second substep of method (1.2) for $n=0,(2.6),(2.7 \mathrm{a})$ (for $i=2),(2.10 \mathrm{c}),(2.14)$ and (2.15), we obtain in turn that

$$
\begin{aligned}
\left\|z_{0}-x^{*}\right\| & \leq\left\|y_{0}-x^{*}\right\|+\left\|A_{0}^{-1} F^{\prime}\left(x^{*}\right)\right\|\left\|F^{\prime}\left(x^{*}\right)^{-1} F\left(y_{0}\right)\right\| \\
& \leq\left(1+\frac{v\left(\left\|y_{0}-x^{*}\right\|\right)}{1-\omega_{0}\left(\delta\left\|x_{0}-x^{*}\right\|,\left\|x_{0}-x^{*}\right\|\right)}\right)\left\|y_{0}-x^{*}\right\| \\
& \leq\left(1+\frac{v\left(g_{1}\left(\left\|x_{0}-x^{*}\right\|\right)\left\|x_{0}-x^{*}\right\|\right)}{1-\omega_{0}\left(\delta\left\|x_{0}-x^{*}\right\|,\left\|x_{0}-x^{*}\right\|\right)}\right) g_{1}\left(\left\|x_{0}-x^{*}\right\|\right)\left\|x_{0}-x^{*}\right\| \\
& =g_{2}\left(\left\|x_{0}-x^{*}\right\|\right)\left\|x_{0}-x^{*}\right\| \leq\left\|x_{0}-x^{*}\right\|<r,
\end{aligned}
$$

which shows (2.11b) holds for $n=0$ and $z_{0} \in B\left(x^{*}, r\right)$. Next, we need an estimate on $\left\|B_{0}\right\|$. Using (2.10e), (2.14)-(2.16) we get in turn that

$$
\begin{aligned}
\left\|B_{0}\right\| & \leq\left\|A_{0}^{-1} F^{\prime}\left(x^{*}\right)\right\|\left[\left\|F^{\prime}\left(x^{*}\right)^{-1} F^{\prime}\left(x^{*}\right)\right\|+\left\|F^{\prime}\left(x^{*}\right)^{-1}\left(\left[w_{0}, x_{0} ; F\right]-\left[z_{0}, y_{0} ; F\right]\right)\right\|\right] \\
& \leq\left\|A_{0}^{-1} F^{\prime}\left(x^{*}\right)\right\|\left[1+\omega\left(\left\|w_{0}-z_{0}\right\|,\left\|y_{0}-x_{0}\right\|\right)\right] \\
& \leq\left\|A_{0}^{-1} F^{\prime}\left(x^{*}\right)\right\|\left[1+\omega\left(\left(\delta+g_{2}\left(\left\|x_{0}-x^{*}\right\|\right)\left\|x_{0}-x^{*}\right\|,\left(1+g_{1}\left(\left\|x_{0}-x^{*}\right\|\right)\left\|x_{0}-x^{*}\right\|\right)\right]\right.\right. \\
& \leq b\left(\left\|x_{0}-x^{*}\right\|\right) .
\end{aligned}
$$

Then, from the last substep of method (1.2), (2.7a) (for $i=3$ ), (2.14), (2.16) and (2.17), we have in turn that

$$
\begin{aligned}
\left\|x_{1}-x^{*}\right\| & \leq\left\|z_{0}-x^{*}\right\|+\left\|B_{0}\right\|\left\|A_{0}^{-1} F^{\prime}\left(x^{*}\right)\right\|\left\|F^{\prime}\left(x^{*}\right)^{-1} F\left(z_{0}\right)\right\| \\
& \leq\left(1+\frac{b\left(\left\|x_{0}-x^{*}\right\|\right) v\left(\left\|z_{0}-x^{*}\right\|\right)}{1-\omega_{0}\left(\delta\left\|x_{0}-x^{*}\right\|,\left\|x_{0}-x^{*}\right\|\right)}\right)\left\|z_{0}-x^{*}\right\| \\
& \leq g_{3}\left(\left\|x_{0}-x^{*}\right\|\right)\left\|x_{0}-x^{*}\right\| \leq\left\|x_{0}-x^{*}\right\|<r,
\end{aligned}
$$

which shows (2.11c) for $n=0$ and $x_{1} \in B\left(x^{*}, r\right)$. The induction for (2.11a)-(2.11c) is completed in an analogous way, if we replace $x_{0}, y_{0}, z_{0}, x_{1}$ by $x_{k}, y_{k}, z_{k}, x_{k+1}$, respectively in the previous estimates. Then, from the estimate

$$
\left\|x_{k+1}-x^{*}\right\| \leq c\left\|x_{k}-x^{*}\right\|<r,
$$

where $c=g_{3}\left(\left\|x_{0}-x *\right\|\right) \in[0,1)$, we deduce that

$$
\lim _{k \rightarrow \infty} x_{k}=x^{*}, \quad x_{k+1} \in B\left(x^{*}, r\right) .
$$

The uniqueness part is shown by assuming $y^{*} \in \Omega_{1}$ with $F\left(y^{*}\right)=0$. Define linear operator $T$ by $T=\left[y^{*}, x^{*} ; F\right]$. Using $(2.9 \mathrm{~b})$ and $(2.12)$, we have in turn that

$$
\left\|F^{\prime}\left(x^{*}\right)^{-1}\left(T-F^{\prime}\left(x^{*}\right)\right)\right\| \leq \omega_{0}\left(0,\left\|y^{*}-x^{*}\right\|\right) \leq \omega_{0}\left(0, R_{1}\right)<1,
$$

so $T$ is invertible. If then follows from the identity $0=F\left(y^{*}\right)-F\left(x^{*}\right)=T\left(y^{*}-x^{*}\right)$ that $x^{*}=y^{*}$. 
Remark 2.1. Method (1.2) is not changing if we use the new instead of the old conditions [16]. Moreover, for the error bounds in practice we can use the computational order of convergence (COC) [18]

$$
\xi=\frac{\ln \frac{\left\|x_{n+2}-x^{*}\right\|}{\left\|x_{n+1}-x^{*}\right\|}}{\ln \frac{\left\|x_{n+1}-x^{*}\right\|}{\left\|x_{n}-x^{*}\right\|}}, \quad \text { for each } n=1,2, \cdots,
$$

or the approximate computational order of convergence (ACOC)

$$
\xi^{*}=\frac{\ln \frac{\left\|x_{n+2}-x_{n+1}\right\|}{\left\|x_{n+1}-x_{n}\right\|}}{\ln \frac{\left\|x_{n+1}-x_{n}\right\|}{\left\|x_{n}-x_{n-1}\right\|}}, \quad \text { for each } n=0,1, \cdots,
$$

instead of the error bounds obtained in Theorem 2.1. Notice that the computation of $\xi^{*}$ does not require knowledge of $x^{*}$. In some cases verifying Lipschitz-type conditions also does not require knowledge of solution $x^{*}$. As an example consider $F$ being Fréchet differentiable and satisfying the autonomous differentiable equation $[2,8]$

$$
F^{\prime}(x)=P(F(x))
$$

where $P: \mathscr{B}_{2} \longrightarrow \mathscr{B}_{2}$ is a known continuous function and say $\mathscr{B}_{1}=\mathscr{B}_{2}=\mathbb{R}$. We have $F^{\prime}\left(x^{*}\right)=P\left(F\left(x^{*}\right)\right)=P(0)$, which is known. By choosing for simplicity $[x, y ; F]=$ $\frac{1}{2}\left(F^{\prime}(x)+F^{\prime}(y)\right)$, we can then verify conditions (2.10a)-(2.10e) without knowledge of the solution $x^{*}$. As an example, let $F(x)=e^{x}-1$, then choose $P(x)=x+1$, so that (2.21) is satisfied.

\section{Numerical examples}

The numerical examples are presented in this section. We choose

$$
[x, y ; F]=\int_{0}^{1} F^{\prime}(y+\theta(x-y)) d \theta .
$$

Example 3.1. Looking back at the motivational example, we see that conditions (2.9b)(2.10f) are satisfied, if we choose $x^{*}=0, F^{\prime}(0)=1, \omega_{0}(s, t)=9.0482(s+t)$,

$$
\delta=\max _{-\frac{\pi}{2} \leq x, y \leq \frac{\pi}{2}}|1+[x, y ; F]|=9.0482,
$$

$v(t)=v_{0}(t)=1+9.0482 t, \omega_{1}(s, t)=9.0482 t$ and $\omega(s, t)=16.5263(s+t)$. The parameters are:

$$
r_{1}=0.0121, \quad r_{2}=0.0080=r_{3}=0.0079=r .
$$

We cannot compare this example with earlier ones such as [16], since no computable radius of convergence was given there. But if it was given under the usual Rheinbold and 
Traub-type Lipschitz conditions $[2-6,8,14,17]$, then, the functions and parameters would have been instead

$$
\begin{aligned}
& \bar{\omega}_{0}(s, t)=\bar{\omega}_{1}(s, t)=\bar{\omega}(s, t)=16.5263(s+t), \\
& \bar{v}_{0}(t)=\bar{v}(t)=1+16.5263 t \\
& \bar{\delta}=\max _{\frac{-\pi}{2} \leq x \leq \frac{\pi}{2}}\left|1+\left[x, x^{*} ; F\right]\right|=7.12427
\end{aligned}
$$

leading to

$$
\overline{r_{1}}=0.0070=\bar{r}, \quad \bar{r}_{2}=0.0091, \quad \bar{r}_{3}=0.0078 .
$$

Notice that $\omega_{0}(s, t)<\bar{\omega}_{0}(s, t), \omega(s, t)=\bar{\omega}(s, t), \omega_{1}(s, t)<\bar{\omega}_{1}(s, t), v_{0}(t)=v(t)<\bar{v}_{0}=$ $\bar{v}(t), \delta<\bar{\delta}, \bar{r}<r$, justifying our approach. Notice also that the results in [16] cannot guarantee the convergence of method (1.2), since $F^{\prime \prime}$ does not exist.

Example 3.2. Let $\mathscr{B}_{1}=\mathscr{B}_{2}=C[0,1], D=\bar{U}(0,1)$. Define function $F$ on $D$ by

$$
F(\varphi)(x)=\varphi(x)-\int_{0}^{1} x \theta \varphi(\theta)^{3} d \theta .
$$

Then, the Fréchet-derivative is given by

$$
F^{\prime}(\varphi(\varsigma))(x)=\varsigma(x)-3 \int_{0}^{1} x \theta \varphi(\theta)^{2} \varsigma(\theta) d \theta, \text { for each } \varsigma \in D .
$$

We get that $x^{*}=0, F^{\prime}\left(x^{*}\right)=I, \omega_{0}(s, t)=\frac{3}{4}(s+t), \delta=\frac{5}{2}, \omega(s, t)=\frac{3}{4}(s+t), v_{0}(t)=$ $v(t)=1+\frac{3}{4} t, \omega_{1}(s, t)=\frac{3}{4} t, \omega(s, t)=\frac{3}{2}(s+t), \delta=\frac{5}{2}$. Then, the radius of convergence $r$ is given by

$$
r_{1}=0.2963, \quad r_{2}=0.1823, \quad r_{3}=0.1135=r .
$$

We cannot compare this example with earlier ones such as [16], since no computable radius of convergence was given there. But if it was given under the usual Rheinbold and Traub-type Lipschitz conditions $[2-6,8,14,17]$, then, the functions and parameters would have been instead $\bar{\omega}_{0}(s, t)=\bar{\omega}_{1}(s, t)=\bar{\omega}(s, t)=\frac{3}{2}(s+t), \bar{v}_{0}(t)=\bar{v}(t)=1+\frac{3}{2} t, \bar{\delta}=3$ leading to

$$
\overline{r_{1}}=0.1481, \quad \bar{r}_{2}=0.2607, \quad \bar{r}_{3}=0.0730=\bar{r} .
$$

Notice that $\omega_{0}(s, t)<\bar{\omega}_{0}(s, t), \omega(s, t)=\bar{\omega}(s, t), \omega_{1}(s, t)<\bar{\omega}_{1}(s, t), v_{0}(t)=v(t)<\bar{v}_{0}=$ $\bar{v}(t), \delta<\bar{\delta}, \bar{r}<r$, justifying our approach.

\section{References}

[1] S. Amat, M. A. Hernández And N. Romero, Semilocal convergence of a sixth order iterative method for quadratic equations, Appl. Numer. Mathe., 62 (2012), pp. 833-841.

[2] I. K. Argyros, Computational Theory of Iterative Methods. Series: Studies in Computational Mathematics, Elsevier Publ. Co. New York, 15 (2007). 
[3] I. K. Argyros, A semilocal convergence analysis for directional Newton methods, Math. Comput., 80(273) (2011), pp. 327-343.

[4] I. K. Argyros and S. Hilout, Weaker conditions for the convergence of Newton's method, J. Complexity, 28 (2012), pp. 364-387.

[5] I. K. Argyros And S. Hilout, Computational Methods in Nonlinear Analysis: Efficient Algorithms, fixed point theory and applications, World Scientific, 2013.

[6] I. K. Argyros And H. Ren, Improved local analysis for certain class of iterative methods with cubic convergence, Numer. Algorithms, 59 (2012), pp. 505-521.

[7] J. M. Gutiérrez, A. A. Magreñán and N. Romero, On the semi-local convergence of NewtonKantorovich method under center-Lipschitz conditions, Appl. Math. Comput., 221 (2013), pp. 79-88.

[8] L. V. Kantorovich and G. P. Akilov, Functional Analysis, Pergamon Press, Oxford, 1982.

[9] A. A. Magrenãn, Different anomalies in a Jarratt family of iterative root finding methods, Appl. Math. Comput., 233 (2014), pp. 29-38.

[10] A. A. Magrenãn, A new tool to study real dynamics: The convergence plane, Appl. Math. Comput., 248 (2014), pp. 29-38.

[11] M. S. Petkovic, B. Neta, L. Petkovic and J. Džunič, Multipoint Methods for Solving Nonlinear Equations, Elsevier, 2013.

[12] J. A. Ezquerro, M. A. Hernández and N. Romero, Aproximación de soluciones de algunas ecuaciones integrales de Hammerstein mediante métodos iterativos tipo Newton, XXI Congreso de Ecuaciones Diferenciales y Aplicaciones (XI Congreso de Matemática Aplicada), 2009.

[13] J. A. Ezquerro, M. A. Hernández and N. Romero, Aproximación de soluciones de algunas ecuaciones integrales de Hammerstein mediante métodos iterativos tipo Newton, XI Congreso de Ecuaciones Diferenciales y Aplicaciones (CEDYA), Universidad de Castilla-La Mancha, 2009, pp. 1-8.

[14] W. C. Rheinboldt, An adaptive continuation process for solving systems of nonlinear equations, Mathematical Models and Numerical Methods, 1978, pp. 129-142.

[15] J. R. Sharma AND P. K. GuHA, An efficient fifth order method for systems of nonlinear equations, Comput. Math. Appl., 67 (2014), pp. 591-601.

[16] J. R. Sharma And D. Kumar, A novel Traub-Steffensen-Chebyshev composition with applications, Appl. Math. Comput., (To appear).

[17] J. F. Traub, Iterative Methods for the Solution of Equations, AMS Chelsea Publishing, 1982.

[18] S. WeErakoon And T. G. I. Fernando, A variant of Newton's method with accelerated thirdorder convergence, Appl. Math. Lett., 13 (2000), pp. 87-93. 